\title{
An alternative proof of Wigner theorem on quantum transformations based on elementary complex analysis
}

\author{
Amaury Mouchet \\ Laboratoire de Mathématiques et de Physique Théorique \\ Université François Rabelais de Tours — CNRS (UMR 7350) \\ Fédération Denis Poisson \\ Parc de Grandmont 37200 \\ Tours, France.
}

\begin{abstract}
According to Wigner theorem, transformations of quantum states which preserve the probabilities are either unitary or antiunitary. This short communication presents an elementary proof of this theorem that significantly departs from the numerous ones already existing in the literature. The main line of the argument remains valid even in quantum field theory where Hilbert spaces are non-separable.
\end{abstract}

Keywords: Wigner theorem, symmetry, unitary representations

Highlights: A new elementary proof of Wigner theorem on quantum transformations is presented. It relies on substantially different hypothesis from the previous proofs. It allows a straightforward generalisation to non-separable Hilbert spaces.

PACS: $11.30 .-\mathrm{j}$ 03.65.Ta 03.70.+k 03.65.-w

Corresponding author : Amaury Mouchet, mouchet@lmpt.univ-tours.fr, phone: +332.47.36.73.70., fax: 33-2.47.36.69.56.

\section{Preliminaries}

Wigner theorem is a cornerstone of theoretical physics since it encapsulates all the linear structure of quantum transformations, among which the evolution of quantum systems (aside from the measurement process). More precisely, given a Hilbert space $\mathcal{H}$ endowed with a Hermitian product $\langle\chi \mid \psi\rangle$

Wigner, in the early 30's (Wigner, 1959, Appendix to chap. 20, pp 233-236, for the updated English translation), acknowledged that any transformation $T:|\psi\rangle \mapsto|\psi\rangle^{\mathrm{T}}=$ $T(|\psi\rangle)$ such that

$$
\forall(|\chi\rangle,|\psi\rangle) \in \mathcal{H}^{2}, \quad\left|{ }^{\mathrm{T}}\langle\chi \mid \psi\rangle^{\mathrm{T}}\right|=|\langle\chi \mid \psi\rangle|
$$

is either or

(a) linear $T\left(c_{1}\left|\psi_{1}\right\rangle+c_{2}\left|\psi_{2}\right\rangle\right)=\left(c_{1}\left|\psi_{1}\right\rangle^{\mathrm{T}}+c_{2}\left|\psi_{2}\right\rangle^{\mathrm{T}}\right)$ and unitary $T^{-1}=T^{*}$;

Email address: mouchet@lmpt.univ-tours.fr (Amaury Mouchet) Preprint submitted to Elsevier 
(b) antilinear $T\left(c_{1}\left|\psi_{1}\right\rangle+c_{2}\left|\psi_{2}\right\rangle\right)=c_{1}^{*}\left|\psi_{1}\right\rangle^{\mathrm{T}}+c_{2}^{*}\left|\psi_{2}\right\rangle^{\mathrm{T}}$ and unitary $T^{-1}=T^{*}($ such a map is also called antiunitary).

We shall systematically use the usual Dirac bra-ket notation and, in the above definitions, $\left(c_{1}, c_{2}\right)$ stands for any pair of complex coefficients, $\left(\left|\psi_{1}\right\rangle,\left|\psi_{2}\right\rangle\right)$ is any pair of elements of $\mathcal{H}$. The Hermitian conjugate will be denoted by ()$^{*}$ and therefore, if $z$ is just one complex number, $z^{*}$ stands for its complex conjugate.

Since Wigner's original work that pertained to the representation of the rotation group, many proofs and generalizations have been proposed whose levels of rigour are not necessarily correlated to their length but, rather, vary depending on the concern of their author. See for example (Simon et al., 2008) and references therein to which I shall add (Fonda and Ghirardi, 1970, § 1.3.2) and the concise and elegant proof given by (Messiah, 1959, $\S \mathrm{XV}-2$ ). The present work is further added to this list because it appears to be almost a back-of-the-envelope presentation while keeping a level of rigour that is acceptable by physicists (hopefully, little additionnal work on the main key ideas should meet to the requirements of mathematicians as well). Moreover, the majority of the previous proofs, if not all of them, can hardly be transposed to non-separable Hilbert spaces, that is to spaces where a countable orthonormal basis does not exist. Yet, in quantum field theory, such non-separable Hilbert spaces are unavoidable: any continuous canonical transformation or rearrangement of the infinitely many degrees of freedom - that physically describe a renormalisation of the bare particles into the dressed ones like, for instance, when a condensation occurs - requires that the Hilbert space is made of a continuous family of orthogonal Fock spaces and one cannot content one self with the unique Fock space that represents the physical (dressed) particles; see for example (Emch, 1972, §1.1) or (Umezawa, 1993, chap. 3).

The proof presented in the second part of the present article will at first make use of a (at most) countable basis $\left(\left|\varphi_{\nu}\right\rangle\right)_{\nu \in I}$ in $\mathcal{H}$,

$$
\left\langle\varphi_{\nu} \mid \varphi_{\mu}\right\rangle=\delta_{\nu \mu},
$$

but the discreteness of the set $I$ will be just a matter of convenience. The transposition to a continuous (multi-)index is straightforward (we will not use any induction arguments that would prevent such generalization) and physicists are quite used to it. For instance, with an appropriate choice of normalization, the Kronecker symbol in (2) is replaced by a Dirac distribution, the discrete sequences $z=\left(z_{\nu}\right)_{\nu \in I}$ labelled by $\nu$ become regular functions $z: \nu \mapsto z(\nu)$, functions of $z$ become functionals, matrices are turned into operators, etc.

Unlike in many proofs, we shall not suppose a priori that $T$ is bijective. The only additional property will be that $T$ is differentiable twice (just once may be sufficient but we will not try no minimize the requirements on the regularity of the transformation). Let us just mention that such smoothness is a reasonable supposition based on physical grounds. Except during the measuring process, when the number of degrees of freedom involved in the interaction of the system with a measuring device becomes infinite and we know that the superposition principle as well as condition (11) are lost by the transformation on the states induced by the measuring process - , we have never observed any discontinuity nor singularity with respect to the quantum state in a transformation.

To see how this differentiable hypothesis simplifies considerably the proof of Wigner theorem by offering a simple strategy, let us see how this works in the Euclidean case 
where the analogous of the Wigner theorem is known as Mazur-Ulam theorem Mazur and Ulam, 1932, this first version, concerning more generally isometries in real normed vector spaces, was published the following year after Wigner's). Let us suppose that $T$ is a differentiable application in an Euclidean space $\mathcal{E}$ preserving the scalar product "." i.e. such that

$$
\forall(u, v) \in \mathcal{E}^{2}, \quad T(u) \cdot T(v)=u \cdot v .
$$

Then, differentiating (3) with respect to $u$ and $v$ leads to

$$
\forall(u, v) \in \mathcal{E}^{2}, \quad\left(T^{\prime}(u)\right)^{\mathrm{t}} T^{\prime}(v)=1
$$

(" $\mathrm{t}$ " denotes the transposition and " " " the derivative) which shows immediately that the Jacobian matrix $T^{\prime}(v)$ is invertible, independent of $v$ and indeed orthogonal. Since moreover $T(0)=0$ (the scalar product is non degenerate), we have proven that $T$ is necessary a linear orthogonal transformation (it is obviously a sufficient condition).

Under the alternative hypothesis of being surjective, the Mazur-Ulam theorem is also immediate since, then, the orthonormal basis $\left(e_{\nu}\right)_{\nu \in I}$ is mapped to an orthonormal basis $\left(e_{\nu}^{\mathrm{T}}\right)_{\nu \in I}$ on which, for any $v$ in $\mathcal{E}$, we can expand $v^{\mathrm{T}}$ and then,

$$
v^{\mathrm{T}}=\sum_{\nu}\left(e_{\nu}^{\mathrm{T}} \cdot v^{\mathrm{T}}\right) e_{\nu}^{\mathrm{T}}=\sum_{\nu}\left(e_{\nu} \cdot v\right) e_{\nu}^{\mathrm{T}} .
$$

If there were a non-null vector $w$ in $\mathcal{E}$ that were orthogonal to the subspace spanned by $\left(e_{\nu}^{\mathrm{T}}\right)_{\nu \in I}$, then its preimage $T^{-1}(w)$, whose existence is guaranteed by the surjectivity hypothesis, would be the null vector, being a vector orthogonal to any $e_{\nu}$. This is in contradiction with $T(0)=0$. Relation (5) can be written as

$$
T\left(\sum_{\nu}\left(e_{\nu} \cdot v\right) e_{\nu}\right)=\sum_{\nu}\left(e_{\nu} \cdot v\right) T\left(e_{\nu}\right)
$$

which expresses the linearity of $T$ whose orthogonality follows. However, in the Hermitian case, the possible phase factors that may appear in the relation between ${ }^{\mathrm{T}}\left\langle\varphi_{\nu} \mid \psi\right\rangle^{\mathrm{T}}$ and $\left\langle\varphi_{\nu} \mid \psi\right\rangle$ make the proofs following the second line of thought much less straightforward.

\section{The core of the proof}

The invariance property formulated in terms of complex analysis. We shall take advantage of the isomorphism that allows us to identify the Hilbert space $\mathcal{H}$ to the set of sequences of complex numbers $z=\left(z_{\nu}\right)_{\nu \in I}$. More precisely, once given an orthonomal basis $\left(\left|\varphi_{\nu}\right\rangle\right)_{\nu \in I}$, we can map any $|\psi\rangle$ in $\mathcal{H}$ to $z$ with $z_{\nu}=\left\langle\varphi_{\nu} \mid \psi\right\rangle$. The Hermitian product in $\mathcal{H}$ reads

$$
\langle w \mid z\rangle \stackrel{\text { def }}{=} w^{*} z=\sum_{\nu} w_{\nu}^{*} z_{\nu}=\langle\chi \mid \psi\rangle
$$

with $z=\left(\left\langle\varphi_{\nu} \mid \psi\right\rangle\right)_{\nu \in I}$ and $w=\left(\left\langle\varphi_{\nu} \mid \chi\right\rangle\right)_{\nu \in I}$. Any application $T$ in $\mathcal{H}$ can be seen as a either a function $\mathcal{T}(x, y)$ of the sequence of the real part $x=\operatorname{Re} z$ and imaginary 
part $y=\operatorname{Im} z$ of the components of the state, or rather as a function of two independent complex sequences $z$ and $\bar{z}$

$$
T(z, \bar{z}) \stackrel{\text { def }}{=} \mathcal{T}((\bar{z}+z) / 2, \mathrm{i}(\bar{z}-z) / 2)
$$

evaluated at $\bar{z}=z^{*}$. When $\mathcal{T}$ can be differentiated, we can define the derivatives with respect to the complex variables by (Dubrovine et al., 1984, $\S 12.1$, for instance)

$$
\begin{aligned}
\partial_{z} T & \stackrel{\text { def }}{=} \frac{1}{2}\left(\partial_{x}-\mathrm{i} \partial_{y}\right) \mathcal{T} ; \\
\partial_{\bar{z}} T & \stackrel{\text { def }}{=} \frac{1}{2}\left(\partial_{x}+\mathrm{i} \partial_{y}\right) \mathcal{T} .
\end{aligned}
$$

In particular, $T$ is analytic if and only if $\partial_{\bar{z}} T=0$. From the differential of $T$, we get

$$
\partial_{z^{*}}\left(T\left(z, z^{*}\right)\right)=\left(\partial_{\bar{z}} T\right)\left(z, z^{*}\right) .
$$

In the following we will drop the distinction between $z^{*}$ and the variable $\bar{z}$ that may vary independently of $z$ because the continuation of any application $T(z, \bar{z})$ defined by (8) in the domain where $\bar{z} \neq z^{*}$ is unique.

In this language, the invariance condition (11) can be reformulated as follows,

$$
\forall(w, z) \in \mathcal{H}^{2}, \quad\left|\left(T\left(w, w^{*}\right)\right)^{*} T\left(z, z^{*}\right)\right|=\left|w^{*} z\right| .
$$

Therefore we have two possibilities : there exists a real function $\theta$ of the complex variables $\left(w, w^{*}, z, z^{*}\right)$ such that

$$
\begin{array}{ll}
\text { (a) either } & \left(T\left(w, w^{*}\right)\right)^{*} T\left(z, z^{*}\right)=\mathrm{e}^{\mathrm{i} \theta\left(w, w^{*}, z, z^{*}\right)} w^{*} z ; \\
\text { (b) or } & \left(T\left(z, z^{*}\right)\right)^{*} T\left(w, w^{*}\right)=\mathrm{e}^{-\mathrm{i} \theta\left(w, w^{*}, z, z^{*}\right)} w^{*} z .
\end{array}
$$

To understand where these two conditions come from, first divide the both sides of the equality in (11) by $\left|w^{*} z\right|$ when non vanishing (then eventually include these cases by continuity of the transformation $T$ ). Thus, we are led to an equation of the form $|Z|=1$ where $Z$ is a complex number. Then, the argument $\eta$ of $Z$ can take the two solutions $\pm \arccos (\operatorname{Re} Z)$ in $[-\pi, \pi]$ of the equation $\cos \eta=\operatorname{Re} Z$. The two options (a) and (b) correspond to the two forms $\mathrm{e}^{ \pm \mathrm{i} \arccos (\operatorname{Re} Z)}$ that is $Z=\mathrm{e}^{\mathrm{i} \arccos (\operatorname{Re} Z)}$ or $Z^{*}=\mathrm{e}^{\mathrm{i} \arccos (\operatorname{Re} Z)}$.

Permuting $\left(w, w^{*}\right)$ and $\left(z, z^{*}\right)$, then by conjugation we have necessarily

$$
\theta\left(z, z^{*}, w, w^{*}\right)=-\theta\left(w, w^{*}, z, z^{*}\right)
$$

which incidentally shows that $\theta(0,0,0,0)=0$.

Adjusting the phase. The first step of our proof is to redefine the phase of the transformed states by considering

$$
\widetilde{T}\left(z, z^{*}\right)=\mathrm{e}^{\mathrm{i} \alpha\left(z, z^{*}\right)} T\left(z, z^{*}\right)
$$

where $\alpha$ is a real function. Conditions (12) can be written

$$
\begin{array}{ll}
\text { (a) either } & \left(\widetilde{T}\left(w, w^{*}\right)\right)^{*} \widetilde{T}\left(z, z^{*}\right)=\mathrm{e}^{\mathrm{i} \tilde{\theta}\left(w, w^{*}, z, z^{*}\right)} w^{*} z ; \\
\text { (b) or } & \left(\widetilde{T}\left(z, z^{*}\right)\right)^{*} \widetilde{T}\left(w, w^{*}\right)=\mathrm{e}^{-\mathrm{i} \tilde{\theta}\left(w, w^{*}, z, z^{*}\right)} w^{*} z .
\end{array}
$$


with

$$
\tilde{\theta}\left(w, w^{*}, z, z^{*}\right)=\theta\left(w, w^{*}, z, z^{*}\right)+\alpha\left(z, z^{*}\right)-\alpha\left(w, w^{*}\right) .
$$

The latter identity can be used to cancel $\tilde{\theta}\left(0,0, z, z^{*}\right)$ with the choice

$$
\alpha\left(z, z^{*}\right)=-\theta\left(0,0, z, z^{*}\right)=\theta\left(z, z^{*}, 0,0\right) .
$$

Therefore without loss of generality, in the following we can consider that for all $z$

$$
\theta\left(0,0, z, z^{*}\right)=0 .
$$

Differentiation. The second step of the proof is to differentiate the conditions written in (12) with respect to $w^{*}$ and $z$. From (12a), using $\partial_{w^{*}}\left(T\left(w, w^{*}\right)\right)^{*}=\left(\partial_{w} T\left(w, w^{*}\right)\right)^{*}$, we get

$$
\begin{aligned}
& \left(\partial_{w} T\left(w, w^{*}\right)\right)^{*} \partial_{z} T\left(z, z^{*}\right)=\mathrm{e}^{\mathrm{i} \theta\left(w, w^{*}, z, z^{*}\right)}\{1+ \\
& +\mathrm{i} w^{*} \partial_{w^{*}} \theta\left(w, w^{*}, z, z^{*}\right)+\mathrm{i} \partial_{z} \theta\left(w, w^{*}, z, z^{*}\right) z \\
& +\left[\mathrm{i} \partial_{z, w^{*}}^{2} \theta\left(w, w^{*}, z, z^{*}\right)\right. \\
& \left.\left.\quad-\partial_{z} \theta\left(w, w^{*}, z, z^{*}\right) \partial_{w^{*}} \theta\left(w, w^{*}, z, z^{*}\right)\right] w^{*} z\right\} .
\end{aligned}
$$

Because of this last computation, and in particular because of the presence of the Hessian of $\theta$, we assume that the transformation is differentiable twice. When evaluated for $\left(w, w^{*}\right)=(0,0)$, we get

$$
\left(\partial_{z} T(0,0)\right)^{*} \partial_{z} T\left(z, z^{*}\right)=1
$$

since (18) implies $\partial_{z} \theta\left(0,0, z, z^{*}\right)=0$. Then $\left(\partial_{z} T\left(z, z^{*}\right)\right)^{-1}=\left(\partial_{z} T(0,0)\right)^{*}$ appears to be independent of $\left(z, z^{*}\right)$ and unitary. Moreover, because $T(0,0)=0$, we have, for all $z$,

$$
T\left(z, z^{*}\right)=\partial_{z} T(0,0) z
$$

and therefore $z \mapsto z^{\mathrm{T}}=T\left(z, z^{*}\right)$ is linear. The linear operator $\hat{U}$ defined by its matrix elements $\left\langle\phi_{\nu}|\hat{U}| \phi_{\nu^{\prime}}\right\rangle^{\text {def }}=\left(\partial_{z} T(0,0)\right)_{\nu \nu^{\prime}}$ is such that $\hat{U}^{-1}=\hat{U}^{*}$ and the expression (21) can be written as $\left\langle\varphi_{\nu} \mid \psi\right\rangle^{\mathrm{T}}=\sum_{\nu^{\prime}}\left\langle\varphi_{\nu}|\hat{U}| \varphi_{\nu^{\prime}}\right\rangle\left\langle\varphi_{\nu^{\prime}} \mid \psi\right\rangle=\left\langle\varphi_{\nu}|\hat{U}| \psi\right\rangle$, hence $|\psi\rangle^{\mathrm{T}}=\hat{U}|\psi\rangle$ which is the first option offered by Wigner theorem.

The second option comes when differentiating (12b) which just modifies the left-hand side of (19) and the irrelevant sign in front of $\theta$ in the right-hand side. Then we obtain

$$
\left(\partial_{z^{*}} T(0,0)\right)^{*} \partial_{z^{*}} T\left(z, z^{*}\right)=1
$$

and eventually

$$
T\left(z, z^{*}\right)=\partial_{z^{*}} T(0,0) z^{*} .
$$

The transformation $z \mapsto z^{\mathrm{T}}=T\left(z, z^{*}\right)$ is antilinear. An antilinear operator $\hat{A}$ can be defined from its matrix elements $\left\langle\phi_{\nu}\right|\left(\hat{A}\left|\phi_{\nu^{\prime}}\right\rangle\right) \stackrel{\text { def }}{=}\left(\partial_{z^{*}} T(0,0)\right)_{\nu \nu^{\prime}}$ and we have $\left\langle\phi_{\nu} \mid \psi\right\rangle^{\mathrm{T}}=\sum_{\nu^{\prime}}\left\langle\phi_{\nu}\right|\left(\hat{A}\left|\phi_{\nu^{\prime}}\right\rangle\right)\left\langle\phi_{\nu^{\prime}} \mid \psi\right\rangle^{*}=\left\langle\phi_{\nu}\right|\left(\hat{A} \sum_{\nu^{\prime}}\left|\phi_{\nu^{\prime}}\right\rangle\left\langle\phi_{\nu^{\prime}} \mid \psi\right\rangle\right)=\left\langle\phi_{\nu}\right|(\hat{A}|\psi\rangle)$ that is $|\psi\rangle^{\mathrm{T}}=\hat{A}|\psi\rangle$. The relation $\left(\partial_{z^{*}} T(0,0)\right)^{-1}=\left(\partial_{z^{*}} T(0,0)\right)^{*}$ reads $\hat{A}^{-1}=\hat{A}^{*}$. Therefore case (b) implies the second alternative of Wigner theorem. 
Summary. Looking back the proof over one'shoulder, once keeping in mind the simple line of thought used in the real case - the double differentiation of (3) that leads to (4) - , the backbone of the argument in the Hilbert case do not appear much more complicated and can be captured in the following few lines : from the key equations (12), it is straightforward by a double differentiation to obtain (19). Then having redefined the phases of the transformed states in order to get (18), we are immediately led to (20) or (22) then to (21) or (23) respectively.

\section{Acknowledgements}

It is a pleasure to thanks Xavier Bekaert for the substantial improvements he suggested after his careful reading of the first version of this note.

\section{References}

Dubrovine, B.A., Fomenko, A.T., Novikov, S.P., 1984. Modern Geometry - Methods and Applications I : The Geometry of Surfaces, Transformation Groups, and Fields. volume 93 of Graduate texts in mathematics. Springer, New York. Translated from the original Russian (Nauka, 1979) by R. G. Burns.

Emch, G.G., 1972. Algebraic methods in statistical mechanics and quantum field theory. John Wiley and sons, New York.

Fonda, L., Ghirardi, G.C., 1970. Symmetry principles in quantum physics. Theoretical Physics, vol. 1, Marcel Dekker, New York.

Mazur, S., Ulam, S., 1932. Sur les transformations isométriques d'espaces vectoriels normés. C. R. Acad. Sci. Paris 194, 946-948. Available on http://gallica.bnf.fr

Messiah, A., 1959. Mécanique Quantique (2 vol.). Dunod, Paris. English translation by G. M. Stemmer: North-Holland (Amsterdam).

Simon, R., Mukunda, N., Chaturvedi, S., Srinivasan, V., 2008. Two elementary proofs of the Wigner theorem on symmetry in quantum mechanics. Phys. Lett. A 372, 6847-6852.

Umezawa, H., 1993. Advances field theory. Micro, macro, and thermal physics. AIP press, New York.

Wigner, E.P., 1959. Group theory and its application to the quantum mechanics of atomic spectra. Academic Press, New York. Édition américaine enrichie et traduite de l'édition originale allemande de 1931 par J.J. Griffin. 